\title{
PARADIGMAS GEOMÉTRICOS EN EL TRABAJO MATEMÁTICO DE DOCENTES EN FORMACIÓN CONTINUA
}

\author{
JESÚS VICTORIA FLORES SALAZAR \\ Pontificia Universidad Católica del Perú - PUCP. Doctora y posdoctora en Educación \\ Matemática. Directora de la maestría en Enseñanza de las matemáticas de la PUCP. ORCID: \\ 0000-0002-0036-140X E-mail: jvflores@pucp.pe

\section{DAYSI JULISSA GARCÍA-CUÉLLAR} \\ Pontificia Universidad Católica del Perú - PUCP Instituto de Investigación sobre la enseñanza \\ de las matemáticas - IREM (PUCP). Magíster en enseñanza de las matemáticas. ORCID: 0000- \\ 0003-0243-6353. E-mail: garcia.daysi@pucp.pe
}




\section{PARADIGMAS GEOMÉTRICOS EN EL TRABAJO MATEMÁTICO DE DOCENTES EN FORMACIÓN CONTINUA}

El presente artículo, evidencia algunos resultados de una investigación de corte cualitativo realizada en una formación continua de profesores en el dominio de la Geometría. La formación se realizó en dos encuentros y participaron ocho estudiantes de posgrado que son docentes peruanos de Educación Básica Regular - nivel secundario. Para este escrito se analiza el trabajo matemático de dos docentes en dos tareas realizadas en el primer encuentro. Como referencial teórico utilizamos aspectos de Espacio de Trabajo Matemático (ETM), especialmente para identificar los paradigmas de la Geometría. En cuanto a los resultados se observó que los docentes resolvieron las tareas en los paradigmas GI y GII del ETM.

Palabras clave: Geometría. Trabajo Matemático. Paradigmas Geométricos. Formación docente.

\section{PARADIGMAS GEOMÉTRICOS NO TRABALHO MATEMÁTICO DE PROFESSORES EM FORMAÇÃO CONTÍNUA}

Este artigo evidencia alguns resultados de uma pesquisa qualitativa realizada em uma formação contínua de professores no domínio de Geometria. A formação foi efetuada em dois encontros e participaram oito estudantes de pós-graduação que também são professores peruanos de Educação Básica - no nível secundário. Para este escrito, analisa-se o trabalho matemático de dois docentes em duas tarefas realizadas no primeiro encontro. Como referencial teórico, utilizamos aspectos do Espaço de Trabalho Matemático (ETM), especialmente para identificar os paradigmas da Geometria. Com relação aos resultados, observase que os docentes resolveram as tarefas nos paradigmas GI e GII do ETM.

Palavras-chave: Geometria. Trabalho Matemático. Paradigmas Geométricos. Formação de professor.

\section{GEOMETRIC PARADIGMS IN THE MATHEMATICAL WORK OF IN-SERVICE TEACHERS EDUCATION}

The present article shows some results of a qualitative research of an in-service teacher in the domain of Geometry. The program was carried out in two meetings with eight postgraduate students who are Peruvian teachers of Regular Basic Education - secondary level. This paper analyzes the mathematical work done by two teachers in two tasks carried out in the first seminar. As a theoretical reference we use aspects of Mathematical Working Space (MWS), especially to identify geometrical paradigms. As for the results, we observe that the teachers solve the tasks in GI and GII paradigms of the MWS.

Keywords: Geometry. Mathematical Work. Geometric Paradigms. In-service teacher education. 


\section{Introdución}

En el Perú el Proyecto Educativo Nacional-PEN al 2021 (PERÚ, 2007) propone estructurar $\mathrm{y}$ fortalecer la formación inicial y continua de profesores, plantea que ambas formaciones deben estar relacionadas ya que, en el caso de matemática, un profesor debe estar preparado para proponer y desarrollar tareas para que los estudiantes sean capaces de resolver problemas, establecer relaciones entre las diferentes representaciones de objetos matemáticos, etc.

Por otro lado, en Didáctica de la Matemática existen investigaciones relacionadas al dominio de la geometría, sin embargo, las evidencias muestran que aún son escasas las investigaciones en este dominio que se vinculen con la formación de profesores. Por ello, es de nuestro interés analizar tareas, en el sentido de la teoría del Espacio de Trabajo Matemático (ETM), que favorezcan el trabajo matemático de profesores en relación al dominio de la Geometría.

En este sentido, en los trabajos recientes que el grupo de investigación Tecnologías y Visualización en Educación Matemática - TecVEM de la Pontificia Universidad Católica del Perú - PUCP, se están desarrollando investigaciones para analizar tareas en el dominio de la Geometría de tal manera que se han identificado conocimientos matemáticos que movilizan docentes en este dominio. Asimismo, en el marco del desarrollo de proyecto de investigación (2019, DGI-694), se cuenta con avances, como los de Almonacid (2018), Salazar y Carrillo (2019).

En relación a la Geometría, se sabe que, durante el último siglo la Geometría y su enseñanza han sufrido dos grandes transformaciones. En primer lugar, la Geometría dejó de ser, por muchas décadas, un área investigación matemática como lo señala Brousseau (1987) la transposición en la enseñanza quedó en manos de los profesores. En segundo lugar, el uso de tecnología digital ha modificado la dinámica de la prueba ${ }^{1}$ en Geometría (STRAESSER, 2002).

1 Prueba en el sentido de Balacheff (2000, p. 12) "El paso de la explicación a la prueba hace referencia a un proceso social por el cual un discurso que asegura la validez de una proposición cambia de posición siendo aceptada por una comunidad. Esta posición no es definitiva; con el tiempo puede evolucionar simultáneamente con el avance de los saberes en los cuales se apoya. Por otro lado, una prueba puede ser aceptada por una comunidad, pero también puede ser rechazada por otra". 
Es así que, Houdement y Kuzniak $(1999 ; 2006)$ introdujeron para el dominio de la geometría tres paradigmas: geometría I (geometría natural), geometría II (geometría natural axiomática), geometría III geometría axiomática formal). Gracias a estas geometrías, es posible comprender ciertos malentendidos y dificultades encontrados en la enseñanza y en la formación de profesores de matemática (KUZNIAK y RAUSCHER, 2011). Además, se enfatiza la necesidad de una enseñanza que favorezca la articulación entre la geometría y los problemas que surgen del mundo real por medio de tareas de modelización. También enfatiza la necesidad de articular la geometría con otros dominios matemáticos.

Sobre formación continua de profesores André (2000) explica que este tipo de formación es considerada indispensable para el docente tanto para actualizar sus conocimientos y técnicas, como para desarrollar competencias y actitudes.

Esto se evidencia en las investigaciones que se fundamentan en el ETM, como la investigación de Henríquez-Rivas y Montoya-Delgadillo (2016) que se centra en contenidos de geometría euclidiana que vinculan los enfoques sintético y analítico en formación de profesores de nivel secundario. En esta investigación se presenta una situación didáctica que se focaliza en el análisis del ETM de un futuro profesor de matemáticas y en las transiciones entre los diferentes paradigmas de la geometría de esta teoría. En esa misma línea de pensamiento, Gómez-Chacón, Botana, Escribano y Abánades (2016) proponen elementos para organizar un ETM para problemas de lugares geométricos con la interacción de software de geometría dinámica y exploran cómo profesores de matemáticas en formación inicial amplían su concepción de lugares geométricos por medio de la apropiación de las funcionalidades específicas del software en relación con su propia práctica como estudiantes y como futuros profesionales.

En cuanto a la mediación de la tecnología digital el enfoque semiótico-instrumental (ARZARELLO, 2006) y los conceptos de razonamiento figurativo y figuro-discursivo (RICHARD, 2004) han permitido conocer mejor cual es el impacto de la utilización de software en la lógica de la prueba en matemática. Además, García-Cuéllar y Salazar (2019) mencionan que trabajar con tecnologías digitales o no (GeoGebra, lápiz y papel), permite al docente identificar en trabajo de los estudiantes (por medio de las acciones) la movilización o creación de posibles esquemas mediados por las tecnologías. 
Por otro lado, Kuzniak, Tanguay y Elia (2016) muestran que existen estudios dedicados al uso de la tecnología digital en la enseñanza y aprendizaje de la matemática que proponen tareas en dominios específicos. En la misma línea de pensamiento, Santos-Trigo, Moreno-Armella y Camacho-Machín (2016) investigan en relación a la resolución de problemas con el uso de tecnología digital por parte de profesores de matemática de nivel secundario y proponen para ello tareas geométricas abiertas. Los autores desarrollan una forma de evaluar estas tareas con fines didácticos mediante la búsqueda de extensiones o generalizaciones facilitadas por el uso de software. Con el soporte del ETM analizan la posible coordinación entre los planos epistemológico y cognitivo para subrayar la importancia de ofrecer un ambiente de aprendizaje donde los fundamentos epistemológicos/ disciplinarios pueden articularse de manera explícita con los sistemas cognitivos de los sujetos.

\section{Espacio de Trabajo Matemático}

A continuación, presentamos algunos aspectos de la teoría Espacio de Trabajo Matemático (ETM) y centramos nuestra atención en el dominio de la Geometría considerando sus Paradigmas.

En ese sentido, Kuzniak, Tanguay y Elia (2016) consideran que el trabajo matemático que realiza el estudiante le permite la construcción de su propio conocimiento sobre la matemática. Sin embargo, afirman que este proceso es gradual, interactivo y complejo, también sostienen que la evolución de los conocimientos matemáticos dependerá de las tareas propuestas y de las actividades que el estudiante realice para resolverlas.

En relación a las nociones básicas del ETM, en la investigación de Kuzniak, Montoya-Delgadillo y Vivier (2015) presentan las nociones de paradigma, dominio, trabajo matemático y tarea. Paradigma es el conjunto de creencias, técnicas y valores que comparte un grupo científico; dominio matemático es determinado según la naturaleza de los objetos estudiados y de los paradigmas que lo caracterizan, por ejemplo, dominio de geometría, algebra, aritmética, análisis, etc.; trabajo matemático consiste en resolver problemas matemáticos, identificar problemas y organizar contenidos dentro de un dominio específico.

En relación con la tarea, en el ETM los autores explican que, de acuerdo con Sierpinska (citada en KUZNIAK et al., 2016), es considerada como cualquier tipo de problema matemático, con preguntas establecidas de manera explícita y clara, que requiere un tiempo predecible para

\section{plurais}


su resolución. Por otro lado, explican que, en el sentido de Chevallard, una tarea está organizada en tipos de tareas y, posee un conjunto de técnicas y conocimientos matemáticos que la respalda. También, los investigadores indican que en el ETM se articulan los planos epistemológico y cognitivo, a través de las génesis originadas por el trabajo matemático.

Estas génesis son detalladas a continuación: Génesis semiótica: basada en los registros de representación semiótica, "es el proceso asociado a los signos y representamen (o significantes), y que representa la relación dialéctica entre la sintáctica y las perspectivas semánticas sobre los objetos matemáticos, desarrollado y organizado mediante sistemas semióticos de representación" (KUZNIAK, et al., 2016, p.726); Génesis instrumental: "permite hacer a los artefactos operativos mediante los procesos de construcción que contribuyen a alcanzar el trabajo matemático" (KUZNIAK, et al., 2016, p.726) y; Génesis discursiva: "utiliza las propiedades del sistema de referencia teórico para ponerlas al servicio del razonamiento matemático y para una validación no solamente icónica, gráfica o instrumental. En la génesis discursiva de la prueba, las propiedades utilizadas en el razonamiento matemático dan el significado" (GÓMEZ-CHACÓN, KUZNIAK y VIVIER, 2016, p. 10).

Los investigadores señalan que el objeto de la génesis discursiva es la validación en sentido bidireccional, por un lado, un razonamiento discursivo apoyado en las propiedades del referencial teórico y, por el otro, la caracterización de propiedades y definiciones que se deben incluir en el marco de referencia, posteriormente a un tratamiento instrumental o semiótico. Además, Kuzniak y Richard (2014) identifican tres planos verticales en el ETM (ver cuadro 1) cada uno de los cuales está definido por la interacción de dos génesis: semiótica e instrumental [Sem-Ins]; instrumental y discursiva [Ins-Dis] y, semiótica y discursiva [Sem-Dis]. 
Cuadro1 - Génesis y planos verticales

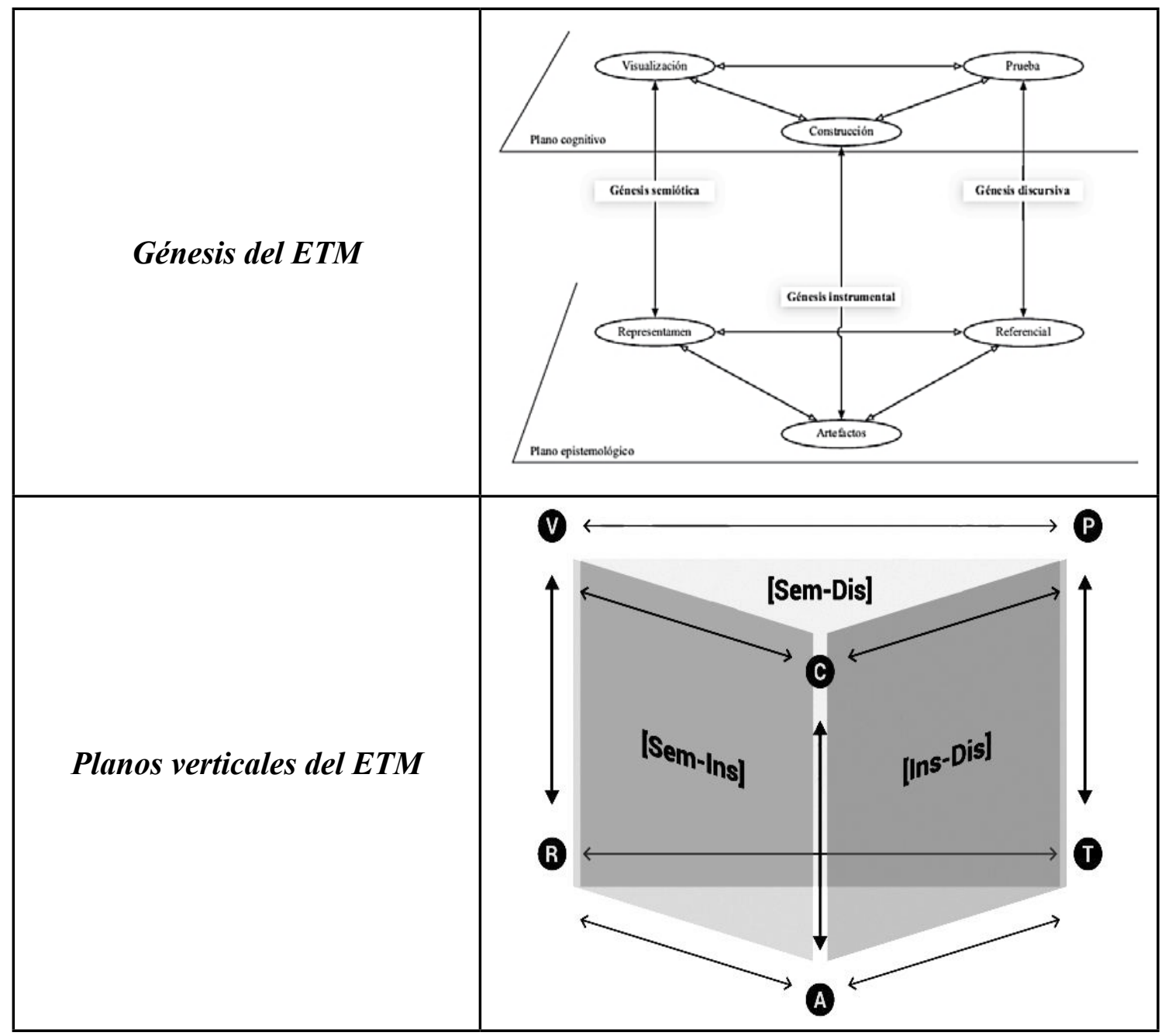

Fuente: Kuzniak y Richard (2014, p. 21) y Kuzniak, Tanguay y Elia (2016, p.726)

Los planos: [Sem-Ins] asociado a una génesis semiótica y a la génesis instrumental. Se observan dos formas de trabajo, una orientada hacia la construcción de los resultados (figuras, gráficos) y la otra hacia la interpretación de los datos brindados por los artefactos; [Ins-Dis] asociado 
a una génesis discursiva de la prueba y a la génesis instrumental y, [Sem-Dis] asociado a las génesis semiótica y discursiva, en el cual se distinguen los razonamientos argumentativos.

Por otro lado, Kuzniak et al. (2016) explican que, para el trabajo matemático en el contexto escolar se tiene que tomar en cuenta las orientaciones curriculares que las instituciones educativas siguen y, que los docentes son los que concretizan en su trabajo en el aula. Además, según las funciones de la institución, el profesor y el estudiante, en el ETM se distinguen tres espacios de trabajo matemático, que son llamados: de referencia, idóneo y personal (GÓMEZ-CHACÓN, et al., 2016, p. 12).

En relación con Houdement y Kuzniak $(1999 ; 2006)$ introdujeron para el dominio de la Geometría tres paradigmas:

Paradigma Geometría Natural - GI: en esta geometría los objetos son “objetos materiales", trazos sobre el papel o trazos digitales cuando se utiliza tecnología digital como softwares, etc., o inclusive maquetas. La técnica, usual en GI es el diseño con el apoyo de instrumentos como: regla graduada, compás, escuadra, transportador, pero también en el plegado, recortado, calcado de papel. Las tareas pueden ser establecidas por la elección de los instrumentos permitidos.

Paradigma Geometría Axiomática Natural - GII: en esta geometría los objetos de estudio son ideales, es decir, es necesario el uso de definiciones, axiomas (propuestos en la geometría euclidiana). Esta geometría se basa en GI conservando una relación con el espacio sensible, es por ello que es llamada por Houdement y Kuzniak (1999) de “axiomática natural”. En esta geometría los problemas deber ser textuales, porque los objetos de este paradigma son las definiciones y los teoremas textuales. Pero los objetos textuales son convencionalmente, por conveniencia, representados por esquemas, con aspecto idéntico a los dibujos de GI, pero sobre los que se debe mirar de diferente manera pues los objetos son conceptuales es por eso que las figuras tienden a substituirse por axiomas y teoremas como objetos de estudio. En este paradigma GII no se utilizan instrumentos materiales, pero si instrumentos intelectuales (razonamiento hipotético-deductivo) que permite construir nuevos conocimientos.

Paradigma Geometría Axiomática Formal - GIII: los objetos también son ideales, el razonamiento hipotético-deductivo es el origen de nuevos conocimientos. La diferencia con GII es que la axiomatización es rigurosa y formal.

El presente artículo se centra en el análisis del trabajo matemático de docentes en el dominio de la Geometría, considerando especialmente los paradigmas. 


\section{Aspectos Metodológicos}

Como la investigación se centra en el análisis de la evidencia de las producciones, estrategias y procesos que realizan profesores de matemática señalamos que la investigación que realizamos es cualitativa (BOGDAN y BIKLEN, 1994). Además, la investigación se desarrolla en coherencia con las fases siguientes:

Primera fase: se realiza la revisión de literatura existente sobre aspectos relacionados al dominio de la geometría, la formación de profesores y la mediación tecnológica, se presentan los aspectos del ETM en el dominio de la Geometría y, la metodología.

Segunda fase: se describe la experiencia de formación y dos tareas que tienen la característica de permitir interpretaciones y el uso de diferentes paradigmas del ETM. Se explicitan las interacciones con los docentes participantes de la formación y se realiza el análisis respectivo.

\section{Experiencia de formación}

La formación fue realizada en el año 2019 en el marco del proyecto de investigación "Modelización matemática y tecnología digital: una propuesta para favorecer el trabajo matemático de profesores en formación continua respecto a la articulación de los dominios de la geometría y del análisis" (DGI 2019-1-0059/ID 694) y constó de dos encuentros, los cuales estuvieron a cargo de dos investigadores de la PUCP/Perú (miembros del grupo TecVEM) y, en los que colaboró también una investigadora de la PUCV/Chile. Además, participaron ocho estudiantes de posgrado que son profesores peruanos de matemática del nivel secundario.

Cuadro 2 - Estructura del primer encuentro ${ }^{2}$

\begin{tabular}{|c|l|}
\hline Tareas & Nombre \\
\hline $\mathbf{1}$ & Paradigmas Geométricos \\
\hline $\mathbf{2}$ & Distancia Mínima \\
\hline
\end{tabular}

Fuente: Elaboración propia

2 Material elaborado por los investigadores de la PUC-SP Dr. Saddo Ag Almouloud y Dra. María José Ferreira da Silva que fue utilizado en una formación de profesores en la PUCP, en el marco del proyecto internacional PEAMATDIMAT, 2015.

\section{plurais}


El cuadro 2 muestra las dos tareas del primer encuentro que presentaremos en este artículo. En la primera tarea llamada "Paradigmas Geométricos" los formadores (investigadores) explicitan aspectos del ETM, en especial los paradigmas del dominio de la Geometría, que servirán de base para la segunda tarea llamada "Distancia Mínima", en la que se solicita a los docentes participantes realizar un análisis matemático y didáctico (asociado a los paradigmas).

En relación con los datos de la formación, estos fueron colectados por medio de fichas de trabajo (docentes participantes), fichas de observación (investigadores) y archivos en GeoGebra. En seguida, presentamos las dos tareas y su respectivo análisis.

Tarea 1: Paradigmas Geométricos

Probar o demostrar que la suma de las medidas de los ángulos internos de un triángulo es igual a $180^{\circ}$. Cuál sería una estrategia de resolución ubicada en el paradigma GI. GIL. GIII?

En GI, se puede confeccionar un triángulo de papel y luego recortar con una tijera los tres ángulos, formando con ellos un semicírculo. En este caso, el docente estará en GI que corresponde a la geometría natural, porque manipula un pedazo de papel de forma triangular y lo recorta.

Por otro lado, si se utiliza un ambiente de representaciones dinámicas (ARD) como es el GeoGebra, que de acuerdo con Salazar y Almouloud (2015) y Salazar, Carrillo, Neira-Fernandez y Montoya-Delgadillo (2019) permite hacer conjeturas e interpretar propiedades que caracterizan a las figuras geométricas representadas.

En ese sentido, para la construcción del triángulo $\mathrm{ABC}$ se puede utilizar distintas herramientas del GeoGebra como, por ejemplo, la herramienta “ángulo” para medir los tres ángulos (ver figura 1). 
Figura 1 - Solución propuestas en el paradigma GII

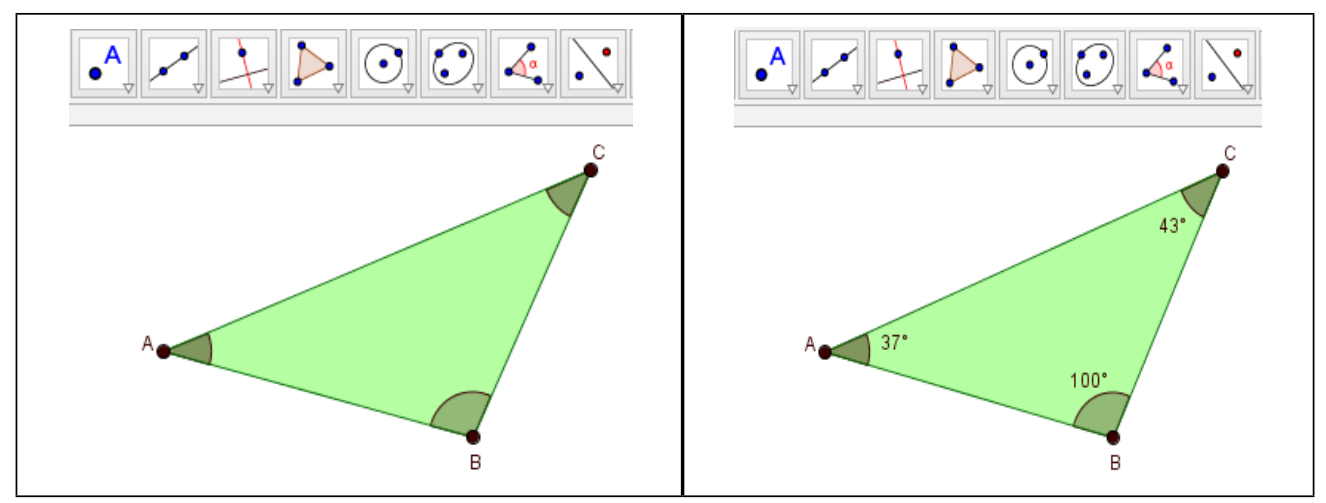

Fuente: Material de la formación

También es posible utilizar la función arrastre para cambiar el triángulo de posición y las medidas (proporcionalmente) de la longitud de sus lados y observar que la suma de las medidas de los ángulos internos es siempre $180^{\circ}$ (ver figura 2).

Figura 2 - Utilizando arrastre y medida del GeoGebra

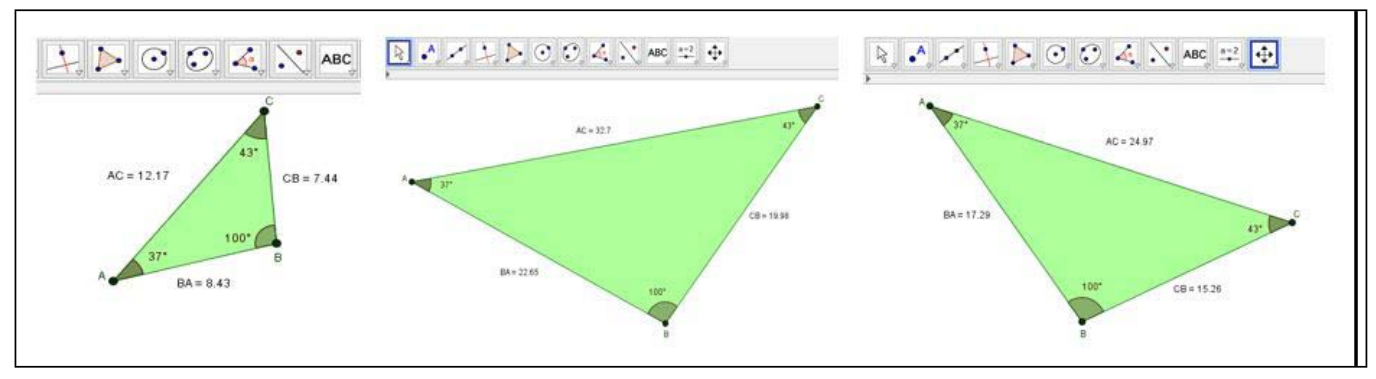

Fuente: Material de la formación

Además, otra posibilidad es comparar su resultado con el resultado de sus colegas, etc. en este caso el docente está en el paradigma GII, pues comprueba el resultado empíricamente en la comparación con los resultados de otros docentes.

\section{Plullais Salvador, v. 5, n. 2, p. 58-77, mai./ago. 2020}


En el caso (ver figura 3), en la que también se utiliza GeoGebra, se traza una recta paralela en uno de los lados y se señala que las retas paralelas determinan ángulos alternos internos congruentes para probar que la suma de las medidas de los ángulos internos de un triángulo es igual a $180^{\circ}$, este trabajo matemático también estaría en el paradigma GII, pues se traza una recta paralela se utiliza la congruencia de los ángulos alternos internos y realiza deducciones en base a estos trazos auxiliares realizados en la misma figura.

Figura 3 - Otra solución en el paradigma GII

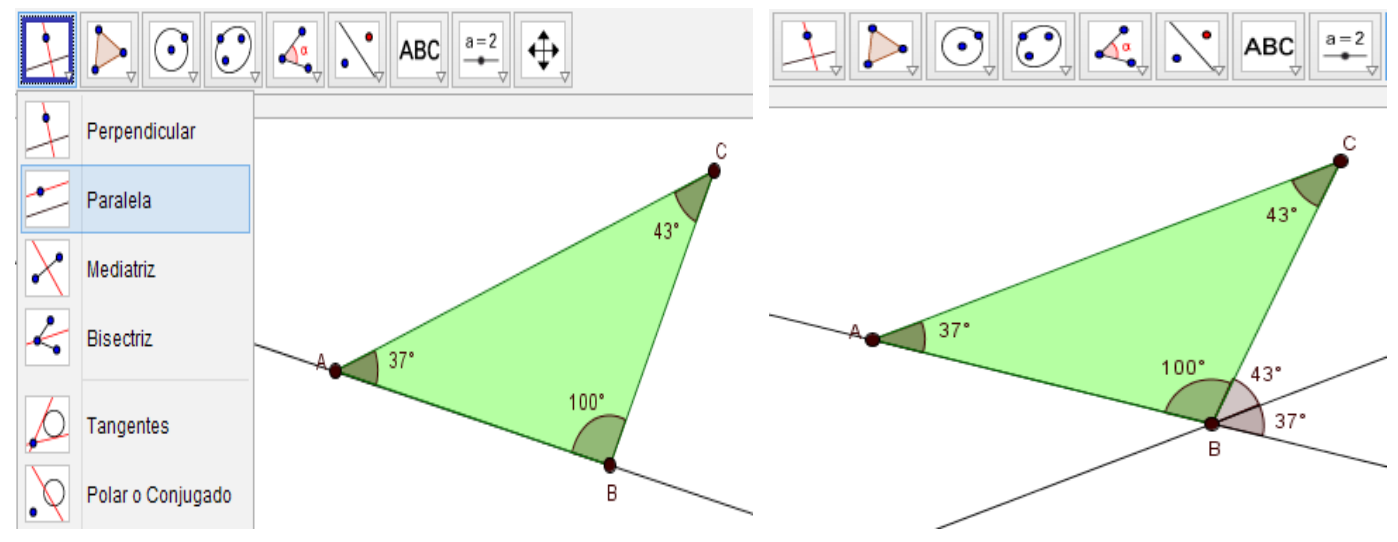

Fuente: Material de la formación

En el caso de realizar una demostración basada en un sistema axiomático de referencia entonces el trabajo matemático se encontraría en el paradigma GIII.

Después de presentada esta tarea, se realizó una discusión didáctica de cada paradigma, en la que los docentes expresaron que justificaciones en los paradigmas GI y GII les son más familiares y son las que generalmente utilizan al enseñar este tema contenido, sin embargo, expresaron que resolver esa tarea en el paradigma GI les resulta un poco difícil.

Los docentes expresaron también, que sus estudiantes de nivel secundario (12 a 15 años), cuando resuelven este tipo de tareas realizan su trabajo matemático, por lo general en los paradigmas GI o GII. 
En seguida, presentamos la tarea "Distancia Mínima" en la que se pide, a los docentes participantes, hacer un análisis matemático y didáctico. Cabe resaltar que la reflexión didáctica fue realizada con la intervención de docentes e investigadores que participaron en la formación.

Tarea 2: Distancia Mínima

Los paralelogramos $A B C D$ y $L M N O$ de la siguiente figura son tales que $A B=L M$.

a) ¿Los dos paralelogramos tienen la misma medida de área?

b) ¿Los dos paralelogramos tienen el mismo perímetro?

Justifique sus respuestas y realice un análisis matemático y didáctico de la tarea.

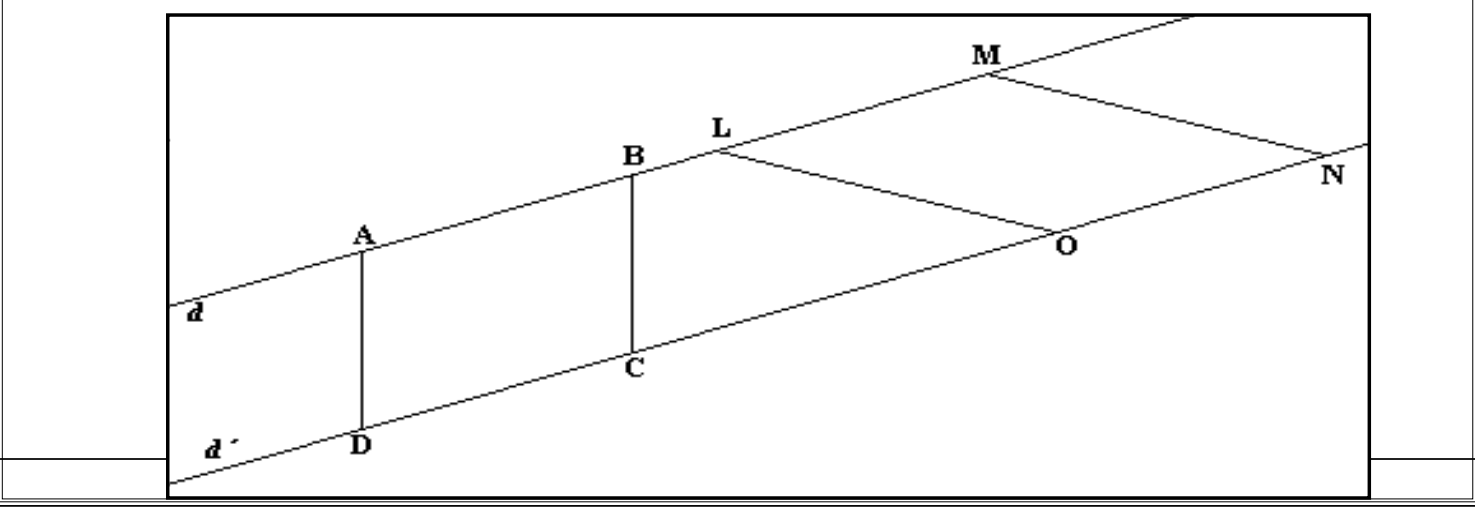

Fuente: Material de la formación

A continuación, presentamos, el desarrollado de la tarea 2. Tomamos como ejemplo el caso de los docentes que denominamos D1 y D2.

El docente D1 basado en la figura dada, realizó la Figura 4 que se muestra a continuación: 
Figura 4 - Tarea realizada por el docente D1

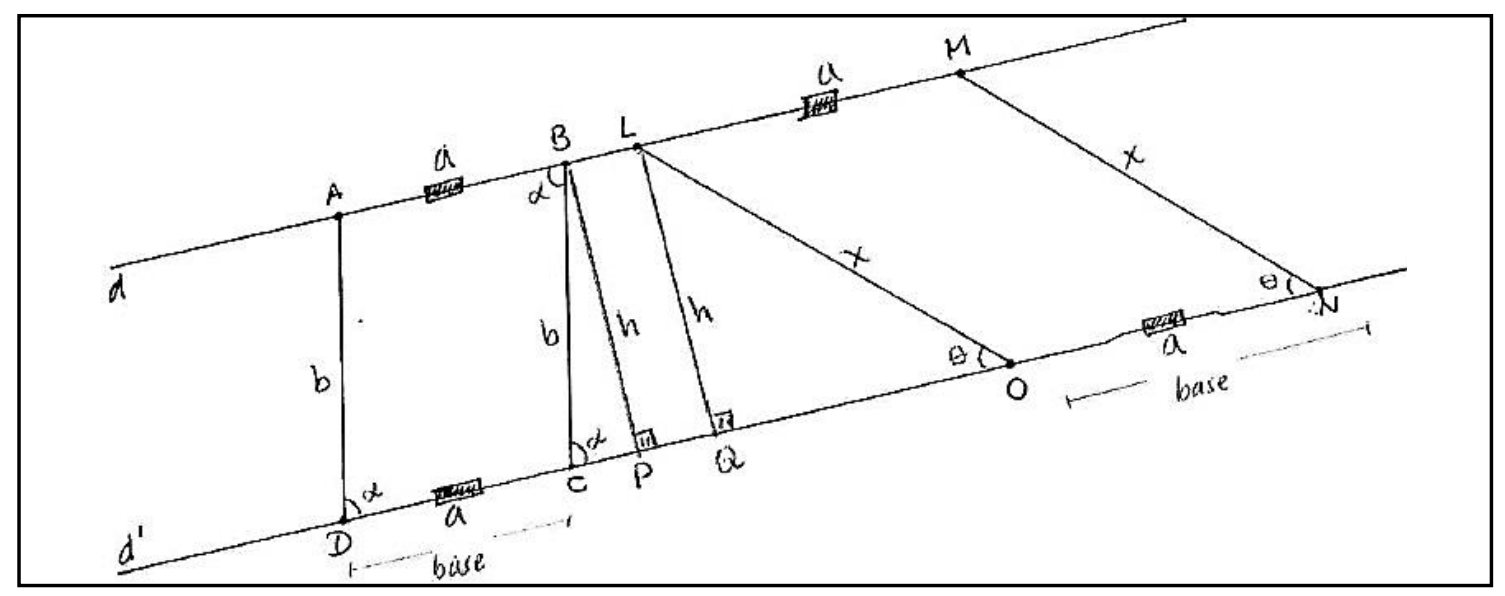

Fuente: Material de la formación

Dadas las condiciones de la tarea los paralelogramos ABCD y LMNO (ver figura 4) tienen la misma medida de área, el docente D1 en la misma figura muestra que moviliza conocimientos matemáticos de congruencia de triángulos, segmentos, ángulos, etc. Lo que evidencia que el trabajo matemático del docente se encuentra en el paradigma GII porque utilizó propiedades y el discurso matemático se encuentra en la misma figura.

En relación con área y perímetro, en la figura 5, se muestra el docente se basa en el enunciado de la tarea y en el discurso que D1 elaboró en la figura para explicar que la medida de las áreas de los paralelogramos $\mathrm{ABCD}$ y $\mathrm{LMNO}$ son iguales. 
Figura 5 - Medida de área realizada por el docente D1

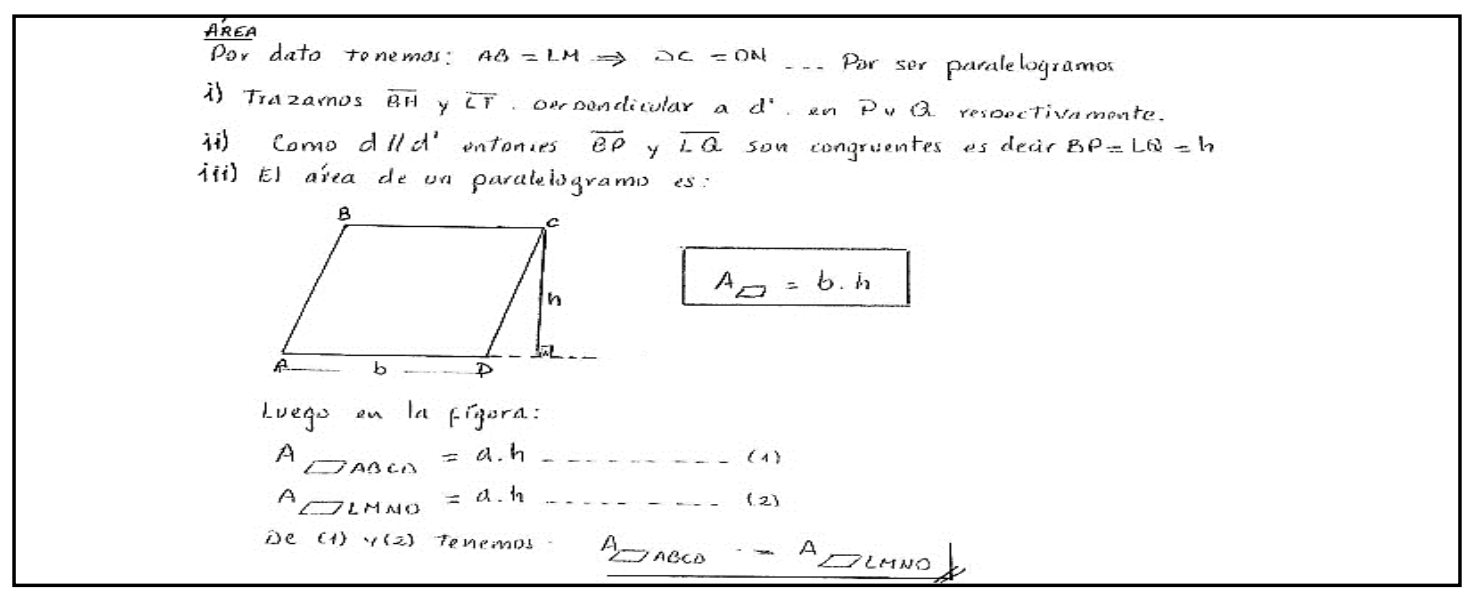

Fuente: Material de la formación

Con relación al perímetro (ver figura 6) el docente D1 con base en el trabajo matemático anterior y utilizando representaciones algebraicas y propiedades de ángulos y afirma que los perímetros no tienen la misma medida.

Figura 6 - Trabajo matemático del docente D1

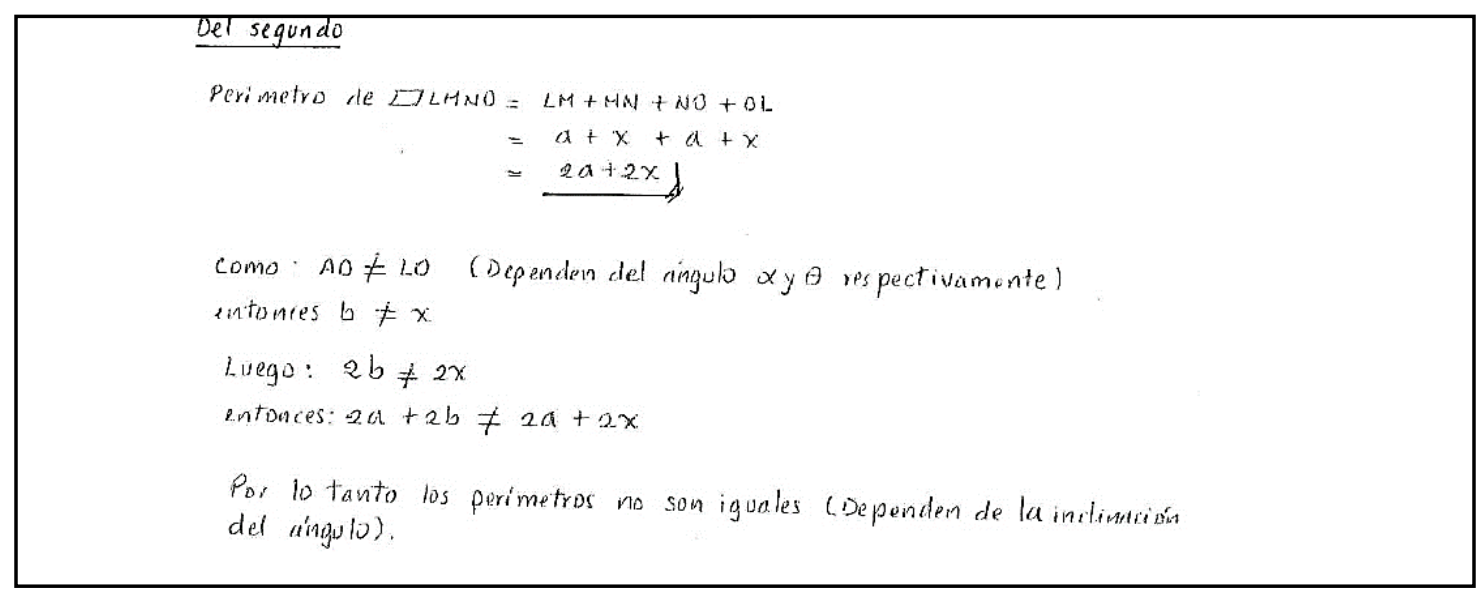

Fuente: Material de la formación

\section{plullais salvador, v. 5, n. 2, p. 58-77, mai./ago. 2020}


Observamos que en el trabajo matemático de D1 realiza validaciones deductivas y por el discurso que realiza el docente su validación correspondería al paradigma GII. A continuación, presentamos el trabajo matemático realizado por el docente D2.

En la figura 7 se muestra el trabajo matemático realizado por el docente D2 y la transcripción de lo escrito por él.

Figura 7- Parte a) Trabajo matemático realizado por el docente D2

\section{En el paradigma GI:}

Para este nivel procedemos de la siguiente manera, respecto al área: Se puede recortar el paralelogramo ABCD (regular) y llevar sobre el paralelogramo LMNO. Se realiza un nuevo recorte, se traslada una región triangular y se comprueba que el área del paralelogramo $\mathrm{ABCB}$ es igual al área del paralelogramo LMNO.

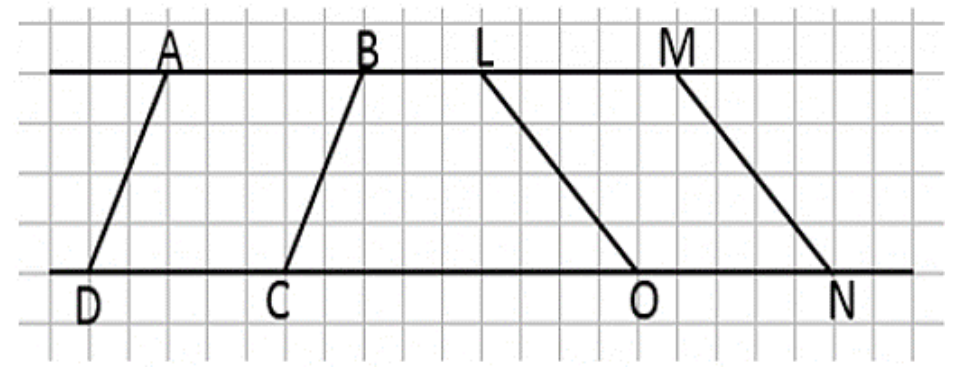

Fuente: Material de la formación

Después, el docente sugiere realizar un nuevo recorte con el fin de trasladar la región triangular y comprobar que ambas figuras poseen la misma medida de área. En cuanto al perímetro, parte b) el docente explica que: "En relación al perímetro, después de realizar el corte se comparan los paralelogramo, encontrándose que y pero, y por lo tanto el perímetro es menor que el perímetro ". Esta afirmación la realiza basado en los conocimientos matemáticos que moviliza en el paradigma GI. 
Sin embargo, observamos que para que el trabajo matemático del docente se considere configurado en GI, su justificación debería basarse en la figura construida sin movilizar otros conocimientos matemáticos, como se evidencia en su justificación. Por ello, se evidencia que el trabajo matemático del docente D2 se encuentra en el paradigma GII.

Después de la intervención de los formadores (investigadores), el docente D2 vuelve a resolver la tarea y realiza trazos auxiliares en la figura y valiéndose del uso de cuadrículas, realiza una descomposición de las figuras para justificar que ambas tienen la misma medida de área (ver Figura 7).

Figura 8 - Tarea realizada por D2 en el paradigma GI

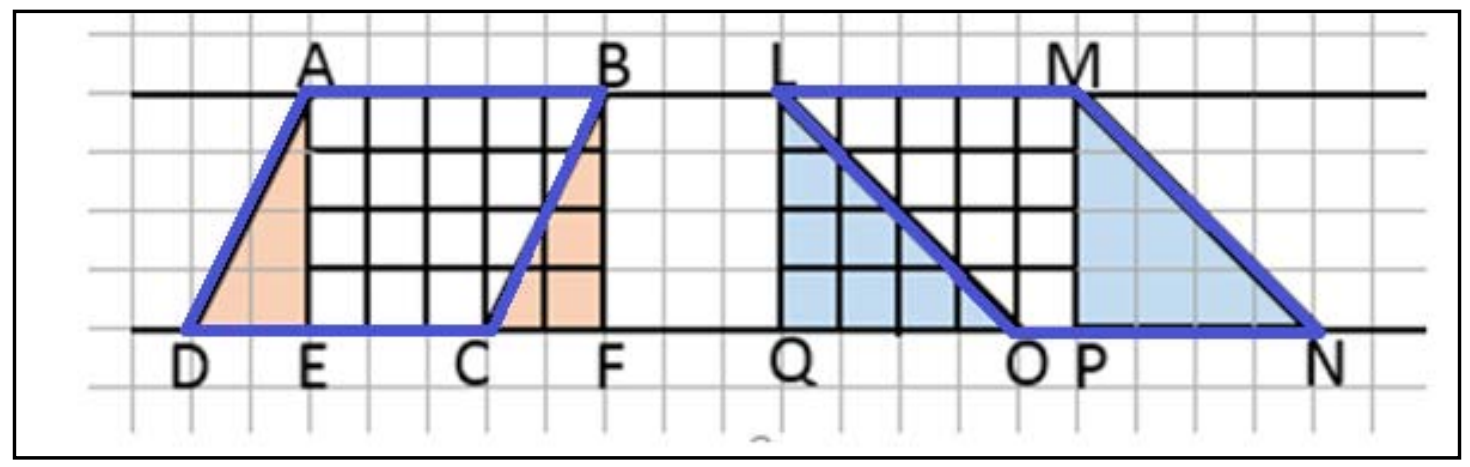

Fuente: Material de la formación

Sin embargo, con este tipo de trabajo matemático el docente D2 no consigue responder, que sucede con el perímetro.

\section{Consideraciones Finales}

En el trabajo matemático del docente D1, se evidencia que su resolución se encuentra en el paradigma GII, porque justifica su trabajo usando propiedades de congruencia de triángulos, definición de cuadriláteros y justifica la medida del área basándose en procedimientos algebraicos.

Lo realizado por el docente D2 muestra que su trabajo matemático se encuentra en el tránsito de los paradigmas GI y GII, es decir que justifica su trabajo basado en la figura (percepción). En

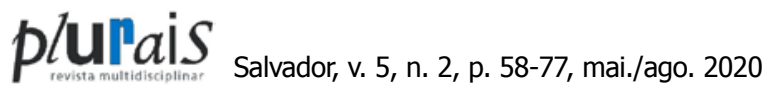


cuanto al grupo de docentes participantes en la formación, podemos afirmar que cinco de los ocho docentes desarrollaron estrategias similares a las presentadas por D2, lo cual los ubica en el tránsito entre los paradigmas GI y GII.

En ese sentido, observamos que tres de los ocho docentes participantes de la formación utilizan la figura como soporte para sus deducciones, lo cual podría evidenciar que desarrollan un trabajo matemático basado fundamentalmente en las propiedades y/o características del objeto matemático representado, lo que significa que al igual que D1 su trabajo matemático se encuentra en el paradigma GII.

En la segunda tarea, parte a), en el trabajo matemático de los docentes se puede observar claramente que en el caso de del docente D1, el discurso matemático que justifica su trabajo, toma como base la figura, sin embargo, la sustenta utilizando propiedades matemáticas. En cambio, para el trabajo matemático del docente D2, la figura es fundamental para su justificación, porque utiliza básicamente la cuadrícula como soporte para determinar la medida del área solicitada en la tarea.

Por otro lado, pensamos que el uso de herramientas del GeoGebra facilita el desarrollo de tareas de manera diferente que, en el ambiente de lápiz y papel, pues favorece la prueba, en el sentido de Balacheff (2000).

Finalmente, en relación al ETM en las tareas analizadas en el artículo observamos que, en varios momentos del trabajo matemático de los docentes, se activan las génesis semiótica, instrumental y discursiva, así como también los planos verticales [Sem- Ins] y [Ins -Dis]. En ese sentido, podríamos afirmar que hubo coordinación entre los planos epistemológico y cognitivo.

\section{Agradecimientos}

Este trabajo fue financiado por la Dirección de Gestión de la Investigación de la PUCP, a través de la subvención DGI 2019-694.

\section{REFERENCIAS}

ALMONACID, A. Modelización de funciones cuadráticas: Espacio de trabajo matemático personal de estudiantes de humanidades. Tesis (Magíster). Enseñanza de las Matemáticas de la Pontificia Universidad Católica del Perú, 2018.

\section{plurais}


ANDRÉ, M. E. D. A. A pesquisa sobre a formação de professores no Brasil - 1990-1998. In: CANDAU, Vera M. (Org.). Ensinar e aprender: sujeitos, saberes e pesquisa. Rio de Janeiro: DP \& A, 83-99, 2000.

ARZARELLO, F. Semiosis as a multimodal process. Revista Latinoamericana de Investigación en Matemática Educativa (RELIME), 9(1), 267-299, 2006.

BOGDAN, R.; BIKLEN, S. Investigação Qualitativa em Educação - uma introdução à teoria e aos métodos. Porto : Porto Editora, 1994.

BROUSSEAU, G. L'enseignement de la géométrie en tant que modèle de l'espace. In G. Brousseau. Théorisation des phénomènes d'enseignement des Mathématiques. Thèse d'état Université de Bordeaux, Bordeaux, France, 447-481, 1987.

GARCÍA-CUÉLLAR, D.; SALAZAR, J. V. F. Estudio de la génesis instrumental del artefacto simbólico simetría axial. TANGRAM - Revista de Educação Matemática, v. 2, n. 3, p. 28-48, 2019.

GÓMEZ-CHACÓN, I.; BOTANA, F.; ESCRIBANO, J.; ABÁNADES, M. The Concept of Locus. Genesis of Personal and Professional Use with Different Tools, Boletim de Educação Matemática - BOLEMA, 30(54), 67-94, 2016.

GÓMEZ-CHACÓN, I., KUZNIAK, A.; VIVIER, L. El rol del profesor desde la perspectiva de los Espacios de Trabajo Matemático, Boletim de Educação Matemática - BOLEMA, 30(54), $1-22,2016$.

HENRÍQUEZ-RIVAS, C.; MONTOYA-DELGADILLO, E. El Trabajo Matemático de Profesores en el Tránsito de la Geometría Sintética a la Analítica en el Liceo. Boletim de Educação Matemática - BOLEMA, 30(54), 45-66, 2016.

HOUDEMENT, C. ; KUZNIAK, A. Un exemple de cadre conceptuel pour l'étude de l'enseignement de la géométrie en formation des maîtres. Educational Studies in Mathematics, 40(3), 283-312, 1999.

HOUDEMENT, C. ; KUZNIAK, A. Paradigmes géométriques et enseignement de la géométrie. Annales de Didactique et de Sciences Cognitives, 11, 175-193, 2006.

KUZNIAK, A., TANGUAY, D.; ELIA, I. Mathematical Working Spaces in schooling: an introduction, ZDM Mathematics Education, 48(6), 721-737, 2016. 
KUZNIAK, A.; RICHARD, P. R. Spaces for Mathematical Work. Viewpoints and perspectives, Revista Latinoamericana de Investigación en Matemática Educativa - RELIME, 17(4-I), 17-28, 2014.

PERÚ, MINISTERIO DE EDUCACIÓN. Proyecto Educativo Nacional al 2021-PEN. Lima, Perú, 2007. Disponible en : http://www.minedu.gob.pe/DeInteres/xtras/PEN-2021.pdf

RICHARD, P. R. L'inférence figurale : un pas de raisonnement discursivo-graphique. Educational Studies in Mathematics. v57, p. 229-263, 2004.

SANTOS-TRIGO, M.; MORENO-ARMELLA, L.; CAMACHO-MACHÍN, M. Problem solving and the use of digital technologies within the mathematical working space framework. ZDM Mathematics Education, 48(6), 827-842, 2016.

SALAZAR, J. V. F.; ALMOULOUD, S. A. Registro figural no ambiente de geometria dinâmica. Educação Matemática e Pesquisa,17(5), 927-932, 2015.

SALAZAR, J.V.F.; CARRILLO, F., NEIRA-FERNANDEZ, V.; MONTOYA-DELGADILLO, E. Dominios de la Geometría y del Análisis y su articulación por medio de la modelización y la tecnología digital. En XV Conferencia Interamericana de Educación Matemática, Medellín: Universidad de Medellín, 1-8, 2019.

SALAZAR, J.V.F.; CARRILLO, F. Espacio de Trabajo Matemático Personal de profesores en relación a la función definida por tramos. Uni-pluriversidad, 19(2), 144-160, 2019.

STRAESSER, R. Cabri-géomètre: Does dynamic geometry software (DGS) change geometry and its teaching and learning? International Journal of Computers for Mathematical Learning, 6(3) 319-333, 2002.

Recebido em: 06 de junho de 2020

Inserido em: 10 de agosto de 2020.

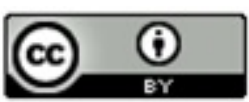

Esta obra está licenciada com uma Licença Creative Commons Atribuição 4.0 Internacional.

\section{plurais}

